\title{
Regular monitoring of breast-feeding rates: feasible and sustainable. The Emilia-Romagna experience
}

\author{
Simona Di Mario ${ }^{1, *}$, Silvana Borsari ${ }^{2}$, Eleonora Verdini ${ }^{3}$, Sergio Battaglia $^{3}$, Luca Cisbani ${ }^{3}$, \\ Stefano Sforza ${ }^{3}$, Chiara Cuoghi ${ }^{4}$ and Vittorio Basevi ${ }^{1}$ \\ ${ }^{1}$ SaPeRiDoc-Documentation Centre on Perinatal and Reproductive Health, Primary Care Service, Regional Health \\ Authority of Emilia-Romagna, Viale Aldo Moro 21, 40127 Bologna, Italy: ${ }^{2}$ Primary Care Service, Regional Health \\ Authority of Emilia-Romagna, Bologna, Italy: ${ }^{3}$ Information Technology Service, Regional Health Authority of \\ Emilia-Romagna, Bologna, Italy: ${ }^{4}$ Local Health Authority, Ferrara, Italy and APEC (Association of Community Pediatricians)
}

Submitted 28 February 2017: Final revision received 28 March 2017: Accepted 3 April 2017: First published online 9 May 2017

\begin{abstract}
Objective: An efficient breast-feeding monitoring system should be in place in every country to assist policy makers and health professionals plan activities to reach optimal breast-feeding rates.

Design/Setting/Subjects: From March to June 2015, breast-feeding rates at 3 and 5 months of age were monitored in Emilia-Romagna, an Italian region, using four questions added to a newly developed paediatric immunization database with single records for each individual. Data were collected at primary-care centres. Breast-feeding definitions and $24 \mathrm{~h}$ recall as recommended by the WHO were used. Direct age standardization was applied to breast-feeding rates. Record linkage with the medical birth database was attempted to identify maternal, pregnancy and delivery factors associated with full breast-feeding rates at 3 and 5 months of age.

Results: Data on breast-feeding were collected for 14044 infants. The mean regional full breast-feeding rate at 3 months was $52 \%$; differences between local health authorities ranged from 42 to $62 \%$. At 5 months of age, the mean regional full breast-feeding rate dropped to $33 \%$ (range between local health authorities: 26 to $46 \%$ ). Record linkage with the birth certificate database was successful for $93 \%$ of records. Total observations more than doubled with respect to the previous regional survey.

Conclusions: The new monitoring system implemented in 2015 in EmiliaRomagna region, totally integrated with the immunization database, has proved to be feasible, sustainable and more efficient than the previous one. This system can be a model for other regions and countries where the vast majority of mothers obtain vaccinations from public health facilities and that already have an immunization database in place.
\end{abstract}

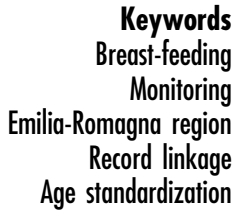

Exclusive breast-feeding up to about 6 months of life and continued breast-feeding thereafter up to 2 years of age and beyond is recommended by the WHO, UNICEF, professional associations, and national and supranational institutions $^{(1-4)}$. Research consistently confirms the association between exclusive breast-feeding and better health, with a dose-response gradient: lower rates of breast and ovarian cancer are reported in breast-feeding mothers, as well as reduced risk of autoimmune, chronic and acute infectious diseases in breast-fed children ${ }^{(5-8)}$. Cost savings for the health system in high-income countries are also reported, due to reduced hospital admission rates, use of drugs and working days lost for caring of sick children $^{(8-14)}$. Finally, better cognitive performances in breast-fed children, and women's empowerment, are thought to possibly contribute to a richer and better social environment $^{(15,16)}$.

Given all of the above, one would expect an efficient breast-feeding monitoring system to be in place in every country to provide policy makers, health professionals and the public at large with reliable and periodically updated breast-feeding rates.

This is not the case, especially in high-income countries ${ }^{(5,17)}$. Annual reports on breast-feeding rates are available only for the USA, Sweden and, starting from 2015, the $\mathrm{UK}^{(18-20)}$. Other countries provide data on a wider time interval: Italy every 5 years ${ }^{(21)}$ and Norway every 8 years $^{(22)}$; Australia $^{(23)}$ and Canada $^{(24)}$ collect data 
on a more erratic basis. Moreover, monitoring systems are rarely integrated into routine health-care systems and often require huge efforts and resources: telephone ${ }^{(18,23)}$ and postal surveys ${ }^{(22,25)}$ are cumbersome and rarely provide a good coverage of the target population, often missing immigrant families. Sometimes, data are collected several months after the end of the breast-feeding period, thus hindering their validity (recall bias) ${ }^{(21,24)}$. To our knowledge there are only two Western countries that regularly monitor breast-feeding rates using a system integrated into routine health care: Sweden and the UK. In Sweden, data are routinely collected during infant visits to the health centre scheduled at 1 week, 2, 4, 6, 9 and 12 months of age, attended by virtually all infants. Aggregate data are thereafter sent to a central system for analysis and annual reporting ${ }^{(19)}$ (Ellen Lundqvist, personal communication, March 2017). In the UK, the long tradition of quinquennial national infant feeding surveys (postal and online) was discontinued in 2010, having acknowledged that it was no longer sustainable ${ }^{(26)}$. From 2015, breast-feeding data are collected from local health authorities during the child health visits scheduled at 6-8 weeks ${ }^{(20)}$.

In Italy, breast-feeding data are monitored every 5 years, along with other health information, using questionnaires administered during a face-to-face interview ${ }^{(21)}$. From 1999 in Emilia-Romagna, a region with 4420000 inhabitants situated in the north-east of Italy, regional data on infant feeding were collected every 3 years, using a paper or an electronic questionnaire (the latter only in 2011) administered at primary-care centres during infant immunization at a given time of the year (the survey usually lasted 6 weeks). In Emilia-Romagna, paediatric vaccines are provided free of charge to all infants at primary-care centres. Appointments are based on national vaccine schedules, the first traditionally being at 3 months of age and the second at 5 months of age (until new arrangements will be made with the introduction of new vaccines).

The objective of the present paper is to describe a new breast-feeding monitoring system implemented in EmiliaRomagna starting from 2015, completely integrated into the routine health-care system, that allows collection of breast-feeding data on an annual basis and that can be linked with other current health databases to provide more information. This system, quite unique with respect to other breast-feeding monitoring systems, can be used as a model if shown to be sustainable.

\section{Methods}

In Emilia-Romagna, up until 2014, paediatric immunization data were provided from primary-care centres to the regional health information and statistics centre as aggregate data. From 2015, a new health database with single records for each individual, containing data on paediatric immunization, was developed and implemented. Health workers at primary-care centres collect and enter data in an electronic form during the immunization visit: along with a set of variables related to demographic data of the child and specific to the vaccines applied, a set of four yes/no questions has been added to assess breastfeeding at the first and second appointment (Box 1). Based on the WHO recommendations ${ }^{(27)}$, the four questions have a recall period of $24 \mathrm{~h}$ and can be used to define mutually exclusive breast-feeding categories: exclusive breast-feeding, predominant breast-feeding (exclusive and predominant breast-feeding together constitute full breastfeeding), complementary feeding and non-breast-feeding (Box 2).

From 16 March to 30 June 2015, guardians of infants aged 61-90d (first immunization visit) and 121-150 d (second immunization visit) were interviewed about infant

Box 1 Four questions used to collect data on infant feeding

In the last $24 \mathrm{~h}$, your baby has received:

- Breast milk?

- Water (including sweetened water), teas, infusions, fruit juice or other non-nutritive liquids, including oral rehydration salts (ORS) solution?

- Formula milk, cow's milk or others not human milk?

- Solid or semi-solid or liquid food other than human milk?

Box 2 Definition of infant feeding (adapted from European Commission $^{(2)}$ and $\mathrm{WHO}^{(27)}$ )

- Exclusive breast-feeding: the infant has received only breast milk from his/her mother or a wet nurse, or expressed breast milk, and no other liquids or solids with the exception of drops or syrups consisting of vitamins, mineral supplements or medicines.

- Predominant breast-feeding: the infant's predominant source of nourishment has been breast milk. However, the infant may also have received water and water-based drinks (sweetened and flavoured water, teas, infusions, etc.); fruit juice; oral rehydration salts (ORS) solution; drop and syrup forms of vitamins, mineral and medicines; and ritual fluids (in limited quantities). Except for fruit juice and sugar water, no food-based fluid is allowed under this definition.

- Exclusive breast-feeding and predominant breastfeeding together constitute full breast-feeding.

- Complementary feeding: the child has received both breast milk and solid (or semi-solid) food.

- Non-breast-feeding: the child has not received breast milk, including expressed breast milk or from a wet nurse. 
feeding. Both Italian and non-Italian speakers were included if understanding was assured, using a linguistic mediator as needed.

Data were sent from the primary-care centres to the regional health information and statistics centre that checked for duplicate records or implausible data. Infants could be included twice (at 3 and 5 months of age) if they had both their first and second immunization visits during the survey interval; in the case of multiple visits, only data gathered during the first appointment for each immunization session were included in the analysis (i.e. once at 3 months and once at 5 months of age). Breast-feeding rates based on the four WHO categories were calculated separately for infants aged 61-90 d (3 months of age) and for infants aged 121-150 d (5 months of age).

The probability to be breast-fed decreases with age and the age of infants presenting to vaccination can range from 61 to $90 \mathrm{~d}$ and from 121 to $150 \mathrm{~d}$ for the 3rd-month and the 5th-month vaccination, respectively. Therefore, it could be misleading to compare the chance of being breast-fed by place (i.e. districts or local health authorities) or by year, without taking in account the differences in age at the vaccination time. Thus, for each age group ( 3 and 5 months), direct age standardization was applied to breast-feeding rates, taking as a reference population the whole group of infants immunized during the 2015 survey, based on $10 \mathrm{~d}$ subgroups (61-70d, 71-80d and 81-90d for infants 3 months of age; and 121-130d, 131-140 d and 141-150d for infants 5 months of age), thereby allowing comparison between groups of infants with comparable age distribution (within a range of $10 \mathrm{~d}$ ). Mean regional breast-feeding rates at 3 and 5 months of age were calculated; mean breastfeeding rates at local health authority level (corresponding roughly to an administrative province of about 100000 to 350000 inhabitants) were also assessed.

Record linkage using a unique identification number of the infant was attempted with the medical birth database (Fig. 1) to identify factors associated with the outcome of interest (full breast-feeding at 3 and 5 months). Statistical analyses were carried out using the Stata statistical software package version 13.1. In the current paper, we present mean rates of breast-feeding at 3 and 5 months of age at regional and at local health authority level and results of the record linkage process.

\section{Results}

In 2015, during the survey period, 15263 infants received one or more vaccines in Emilia-Romagna region (8459 at 3 months and 6804 at 5 months of age), $51 \%$ of the infants were male and $32 \%$ were born to non-Italian mothers, mostly from Morocco (6\%), Romania (4\%) and Albania ( $4 \%)$. As foreseen, age distribution of infants, based on $10 \mathrm{~d}$ classes, varied between local health authorities at both the first (Fig. 2) and the second vaccination (Fig. 3).

Data on breast-feeding were available for 14044 infants (7861 at 3 months and 6183 at 5 months of age). The mean regional full breast-feeding rate was $52 \%$ (45\% exclusive and $7 \%$ predominant) at 3 months; it dropped to $33 \%$ ( $27 \%$ exclusive and $6 \%$ predominant) at 5 months. Differences between local health authorities were large both at 3 months (range: $42-62 \%$; Fig. 4) and at 5 months of age (range: 26-46\%; Fig. 5).

Record linkage between the immunization database and the medical birth database was successful for 14192 records out of 15263 (93\%), most linkage failures being due to infants born outside the Emilia-Romagna region (872 out of 1071 linkage failures) for which the corresponding birth certificates were not available. Considering only records from the immunization database reporting breast-feeding data, and for which record linkage with the medical birth database was successful, a total of 13177 observations were available. For those records, data retrieved from the medical birth database were complete for most of the analysed variables; in particular, completeness was $100 \%$ for nationality of the mother, maternal education, labour and delivery mode, gestational age at birth and single fetus, but it went down to $96 \%$ for paternal education, to $92 \%$ for maternal overweight/ obesity, and to $84 \%$ for epidural anaesthesia (Table 1).

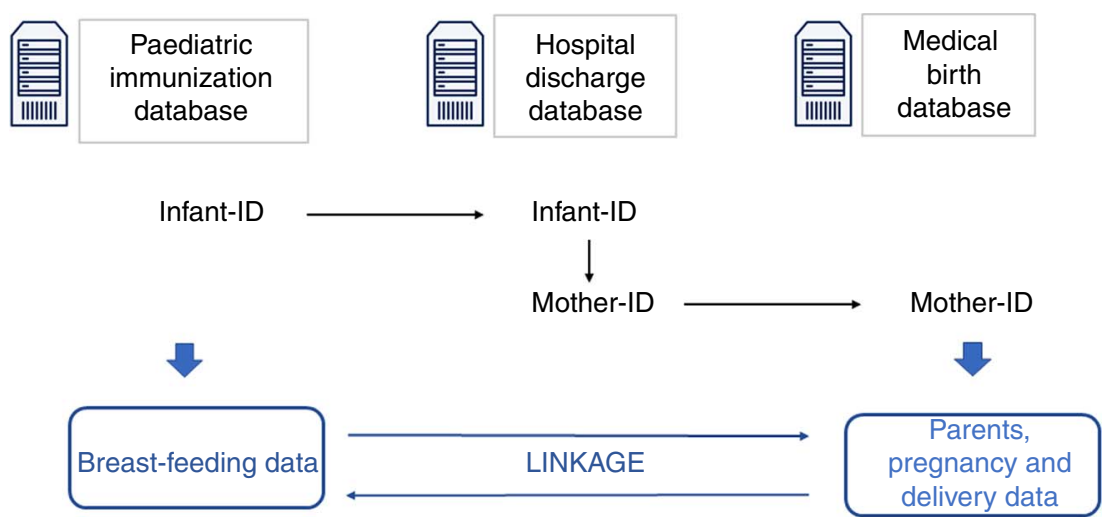

Fig. 1 (colour online) Outline of record linkage between paediatric immunization database and medical birth database (ID, identification number) 




Fig. 2 (colour online) Age distribution ( $\square, 61-70 \mathrm{~d}$; $\square, 71-80 \mathrm{~d}$; $\square, 81-90 \mathrm{~d}$ ) of the 8459 infants receiving the first vaccination (3 months of age), by local health authority, Emilia-Romagna region, Italy, 2015



Fig. 3 (colour online) Age distribution ( $\square, 121-130 \mathrm{~d}$; $\square, 131-140 \mathrm{~d}$; $\square, 141-150 \mathrm{~d}$ ) of the 6804 infants receiving the second vaccination (5 months of age), by local health authority, Emilia-Romagna region, Italy, 2015

Total observations more than doubled with respect to the previous survey: in 2011 breast-feeding information was collected for 6065 infants, whereas in 2015 a total sample of 14044 was observed.

In 2016 the survey was repeated; health professionals agreed to collect data for a longer period, from 16 March to 30 November: data on breast-feeding were collected for 33244 infants (analysis of data in progress).

\section{Discussion}

In Emilia-Romagna, breast-feeding rates at 3 and 5 months of age fall short of the recommended level. Interventions are needed to better support mothers and families and to attain successful breast-feeding, lasting longer, in a much larger number of women. To monitor the impact of such interventions, annually updated local data are necessary. 




Fig. 4 (colour online) Mean breast-feeding rates ( $\square$, exclusive breast-feeding; $\square$, predominant breast-feeding; $\square$, complementary feeding; $\square$, non-breast-feeding; -... , mean regional full breast-feeding rate at 3 months) at the first immunization visit (3 months of age), by local health authority, Emilia-Romagna region, Italy, 2015

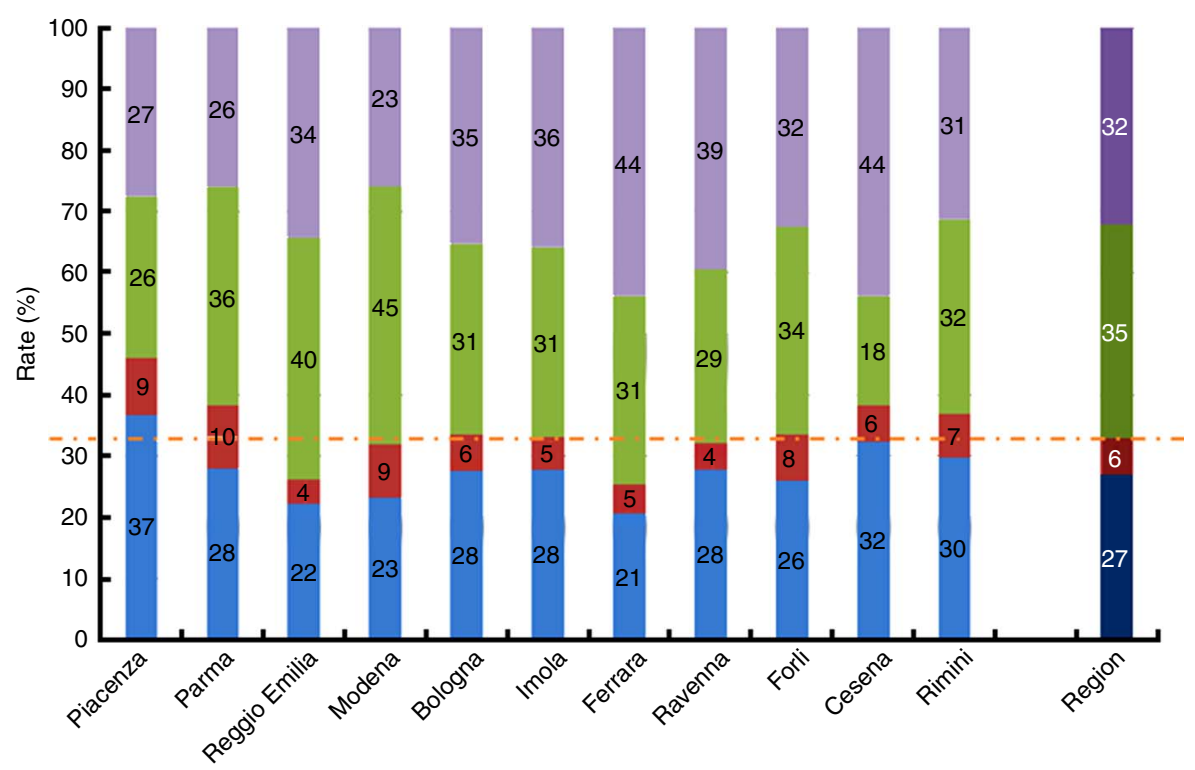

Fig. 5 (colour online) Mean breast-feeding rates ( $\square$, exclusive breast-feeding; $\square$, predominant breast-feeding; $\square$, complementary feeding; $\square$, non-breast-feeding; - - . - mean regional full breast-feeding rate at 5 months) at the second immunization visit (5 months of age), by local health authority, Emilia-Romagna region, Italy, 2015

The new monitoring system to assess breast-feeding rates implemented in 2015 in Emilia-Romagna region has proved to be feasible, sustainable and more efficient than the previous one: observations more than doubled, standardization provided more comparable data between different local health authorities, record linkage with another regional health database has provided insights on local protective and risk factors for successful breastfeeding without need for additional resources, the survey has been repeated in 2016 for a longer period and a new round of data collection is already planned for 2017. Switching from a self-standing questionnaire to a totally integrated system that flows along the immunization database has reduced the workload of the health professionals working in the primary-care centres who are responsible for the breast-feeding survey. No additional personnel were requested, as the survey required only a little extra work (four yes/no answers to be entered online). Health professionals involved in the monitoring system spontaneously proposed to double the survey 
Table 1 Missing data for the 13177 records providing breastfeeding data and for which record linkage was successful, EmiliaRomagna region, Italy, 2015

\begin{tabular}{lrc}
\hline Variable & $n / 13177$ & $\%$ \\
\hline Maternal nationality & 0 & 0.0 \\
Maternal age & 30 & $0 \cdot 2$ \\
Maternal education & 0 & 0.0 \\
Paternal education & 543 & 4.1 \\
Maternal smoking & 110 & 0.8 \\
Antenatal course & 68 & 0.5 \\
Maternal BMI & 1066 & $8 \cdot 1$ \\
Labour and delivery mode & 0 & 0.0 \\
Gestational age at birth & 0 & 0.0 \\
Birth weight & 1 & 0.0 \\
Single fetus & 0 & 0.0 \\
Epidural anaesthesia & 2107 & 16.0 \\
\hline
\end{tabular}

period for the year 2016 (collecting phase already concluded) and for the years to come. Having more observations provides more precise estimates of breast-feeding rates not only at regional level, but also at local health authority level. This is important, as planning and financing local projects to protect, promote and support breastfeeding depends on local health authorities.

Moreover, using age-standardized data for assessing and comparing breast-feeding rates gets rid of possible distortions due to the ample range of ages at which infants receive their first and second immunization: in our region, almost a quarter $(23 \%)$ of infants get their first vaccination between 61 and $70 \mathrm{~d}$ and another quarter (24\%) between 81 and $90 \mathrm{~d}$, with large differences between local health authorities (Fig. 2); a similar dispersion in terms of age is observed also for infants getting the second immunization (Fig. 3). Direct age standardization permits comparisons between different local health authorities (Figs 4 and 5) and, using the same standard population along the years, will allow meaningful comparison during time and assessment of trends. To our knowledge, this is the first time that age-standardized breastfeeding rates have been used.

Finally, the presence of a unique anonymous identification number consistent across different regional health databases allows gathering additional information through record linkage. The Emilia-Romagna regional health system has a rich set of administrative health databases, describing relevant conditions like births, hospital admissions, drug prescriptions, laboratory examinations and deaths ${ }^{(28)}$. Information from regional databases is collected as disaggregated data (one record for each patient). To guarantee privacy, a unique anonymous personal identification number is associated with every single user of the health system. The personal identification number is consistent for each individual across different databases and along time: therefore, through record linkage of different databases it is possible to describe each contact between a citizen and the regional health system during his/her entire life. In 2015 the record linkage of the infant feeding database was made with the medical birth database and it has been successful for most observed cases ( $93 \%$ of the total). In the future, it will be possible to plan linkages with other regional databases: for example, record linkage with the children's hospital discharge database or with the drug prescriptions database could provide insights about the additional costs in terms of health-care consumption of not being breast-fed. Providing only aggregated data does not allow this sort of analyses: in Sweden, for example, data are sent in an aggregated form for all of the twenty-one counties (Ellen Lundqvist, personal communication, March 2017), with the exception of Uppsala and Orebro counties for which single patient data are available ${ }^{(29)}$.

A limitation of our study is that feeding information was incomplete (data available for 14044 out of 15263 immunized infants, $92 \%$ of the total). As this was the first year of implementation of a new monitoring system, we expect more complete data in the future. Moreover, completeness was not satisfactory for all variables of the medical birth database (Table 1): information on paternal education, maternal overweight/obesity and epidural anaesthesia was missing in 4,8 and $16 \%$ of records, respectively (information on epidural anaesthesia was included in the medical birth database for the first time in 2015; completeness is expected to improve in the future).

Finally, even though the use of immunization sessions is a good opportunity to collect data on infant feeding, as paediatric immunizations are exclusively provided at immunization centres in Emilia-Romagna, it excludes infants whose parents do not comply with vaccination schedules. Notwithstanding, we think that this system is the one that can provide the more complete picture of breastfeeding in our region, and in other regions and countries as well. In Emilia-Romagna, infant immunizations are totally missing in about $3 \%$ of the infants ${ }^{(30)}$; thus regional estimates of breast-feeding rates should not be grossly erroneous due to those missing data. It is a common belief that families that are totally against immunization are generally favourable to breast-feeding, but at present we do not have data proving it. It could be of interest, in the future, to conduct a focused analysis, using a different data collection instrument, to study infant feeding attitudes and practices in families that oppose vaccination.

In our opinion, the system described herein, and used in Emilia-Romagna region starting from 2015, can be a model for other Italian regions and other high-income countries that already have in place a set of national health databases, including an immunization database, and for which immunization is universally delivered within a unique health setting. The Emilia-Romagna system has been shown to be feasible and sustainable at no, or very little, additional cost. Comments gathered from our health professionals, working in the immunization centres and collecting and inputting feeding data, reassured us that the new monitoring system is much easier to comply with, as it requires less time, avoids double input of data and has been proposed for use during the entire calendar year. 


\section{Acknowledgements}

Acknowledgements: The authors wish to thank Bruna Borgini, Michela Bragliani and Elena Castelli for administrative support at regional level; APEC (Association of Community Pediatricians) for conceiving and sustaining the monitoring system since 1999; and Adriano Cattaneo for providing suggestions in the phase of analysis and for revising the draft of the paper. They also thank all coordinators for the 2015 survey at local level: Angela Castellana, Carla Ghigini, Roberto Maffi, Anna Maria Milani, Raffaella Pellizzari and Marilena Prazzoli (Piacenza Azienda Unità Sanitaria Locale (AUSL)); Giovanna Bussolati and Icilio Dodi (Parma AUSL); Marina Sparano, Luigi Moscara and Alessandro Volta (Reggio Emilia AUSL); Giulio Sighinolfi and Maria Vezzani (Modena AUSL); Paola Lenzi and Rita Ricci (Bologna AUSL); Paola Caroli and Elisabetta Valenti (Imola AUSL); Flavia Pascoletti and Massimo Cornale (Ferrara AUSL); Luisa Baldi, Giovanna Cappelli, Oriana Gasperoni, Claudia Muratori and Ivonne Zoffoli (ex Ravenna AUSL); Anna Maria Baldoni (ex Forlì AUSL); Antonella Brunelli, Massimo Farneti, Marcella Lombardi and Fosca Marrone (ex Cesena AUSL); Maurizio Bigi and Antonella Mazzocchi (ex Rimini AUSL). Finally, the authors are sincerely grateful to all parents and caregivers who participated to the survey for providing infant feeding information. Financial support: This research received no specific grant from any funding agency in the public, commercial or not-for-profit sectors. Conflict of interest: None. Authorship: S.D.M. and V.B. conceived and planned the paper; S.D.M., S. Borsari, E.V. and C.C. developed the monitoring system; S.D.M. and C.C. coordinated the data collection; S.S., L.C. and S. Battaglia managed the data fluxes and performed the record linkage; S.S. and S.D.M. analysed the data; S.D.M. wrote the first report; all co-authors revised, commented and approved the paper. Ethics of human subject participation: Not applicable.

\section{References}

1. World Health Organization (2003) Global strategy for infant and young child feeding. http://apps.who.int/iris/bit stream/10665/42590/1/9241562218.pdf?ua=1\&ua=1 (accessed February 2017).

2. European Commission, Directorate Public Health and Risk Assessment (2008) EU Project on Promotion of Breastfeeding in Europe. Protection, Promotion and Support of Breastfeeding in Europe: A Blueprint for Action (revised). http://ec.europa. eu/health/ph_projects/2002/promotion/fp_promotion_2002_ frep_18_en.pdf (accessed February 2017).

3. McGuire S (2011) US Dept. of Health and Human Services. The Surgeon General's Call to Action to Support Breastfeeding. US Dept. of Health and Human Services, Office of the Surgeon General. 2011. Adv Nutr 2, 523-524.

4. Italian Ministry of Health (2016) Allattare al seno, un investmento per la vita (Breastfeeding, a lifelong investment). http://www.salute.gov.it/imgs/C_17_opuscoliPoster_ 303_allegato.pdf (accessed February 2017).
5. Victora CG, Bahl R, Barros AJD et al. (2016) Breastfeeding in the 21st century: epidemiology, mechanisms, and lifelong effect. Lancet 387, 475-490.

6. Ip S, Chung M, Raman G et al. (2007) Breastfeeding and maternal and infant health outcomes in developed countries. Evid Technol Assess (Full Rep) 153, 1-186.

7. Eidelman AI, Schanler RJ \& American Academy of Pediatrics (2012) Breastfeeding and the use of human milk. Pediatrics 129, e827-e841.

8. Renfrew MJ, Dyson L, McCormick F et al. (2010) Breastfeeding promotion for infants in neonatal units: a systematic review. Child Care Health Dev 36, 165-178.

9. Bartick MC, Schwarz EB, Green BD et al. (2017) Suboptimal breastfeeding in the United States: maternal and pediatric health outcomes and costs. Matern Child Nutr 13, e12366.

10. Ma P, Brewer-Asling M \& Magnus JH (2013) A case study on the economic impact of optimal breastfeeding. Matern Child Health J 17, 9-13.

11. Smith JP (2013) 'Lost milk?'. J Hum Lact 29, 537-546.

12. Pokhrel S, Quigley MA, Fox-Rushby J et al. (2015) Potential economic impacts from improving breastfeeding rates in the UK. Arch Dis Child 100, 334-340.

13. Rollins NC, Bhandari N, Hajeebhoy N et al. (2016) Why invest, and what it will take to improve breastfeeding practices? Lancet 387, 491-504.

14. Cattaneo A, Ronfani L, Burmaz T et al. (2006) Infant feeding and cost of health care: a cohort study. Acta Paediatr 95, 540-546.

15. Horta BL, Loret De Mola C \& Victora CG (2015) Breastfeeding and intelligence: a systematic review and metaanalysis. Acta Paediatr 104, 14-19.

16. Labbok MH, Smith PH \& Taylor EC (2008) Breastfeeding and feminism: a focus on reproductive health, rights and justice. Int Breastfeed J 3, 8.

17. Bagci Bosi AT, Eriksen KG, Sobko T et al. (2016) Breastfeeding practices and policies in WHO European Region Member States. Public Health Nutr 19, 753-764.

18. Scanlon KS, Li R, Chen J et al. (2014) Estimation of breastfeeding rates in the United States from the National Immunization Survey: the effect of adding a cellular telephone sample of respondents. https://www.cdc.gov/breastfeeding/ data/nis_data/estimation-bf-rates.htm (accessed February 2017).

19. Socialstyrelsen, The National Board of Health and Welfare (2016) Statistics on breastfeeding 2014. https://www. socialstyrelsen.se/Lists/Artikelkatalog/Attachments/20333/ 2016-9-20.pdf (accessed February 2017).

20. Public Health England (2016) Official Statistics. Breastfeeding prevalence at 6 to 8 weeks after birth: annual data. https://www.gov.uk/government/statistics/breastfeeding-at6-to-8-weeks-after-birth-annual-data (accessed February 2017).

21. Socio-demographic and Environmental Statistics Directorate, Istat - National Institute of Statistics (2014) Pregnancy, childbirth and breastfeeding in Italy: Year 2013. https:// www.istat.it/en/files/2014/12/Pregnancy-childbirth-breastfeeding-2013.pdf (accessed February 2017).

22. Øverby NC, Kristiansen og AL, Andersen LF et al. (2008) Spedkost - 6 måneder. Landsomfattende kostholdsundersøkelse blant 6 måneder gamle barn (Norwegian national dietary survey among infants at 6 months). https:// helsedirektoratet.no/publikasjoner/spedkost-6-maneder-land somfattende-kostholdsundersokelse (accessed February 2017).

23. Australian Institute of Health and Welfare (2011) 2010 Australian national infant feeding survey: indicator results. Catalogue no. PHE 156. http://www.aihw.gov.au/publication-detail/?id=10737420927 (accessed February 2017).

24. Gionet L (2015) Breastfeeding trends in Canada: 2011-2012 data. Statistics Canada Catalogue no. 82-624-X. http://www. statcan.gc.ca/pub/82-624-x/2013001/article/11879-eng.htm (accessed February 2017) 
25. Australian Institute of Family Studies (2008) Growing up in Australia: the longitudinal study of Australian children, annual report 2006-2007. http://www.growingupinaustralia.gov.au/ pubs/ar/ar200607/breastfeeding (accessed February 2017).

26. IFS News (2010) The Infant Feeding Survey has been discontinued. http://content.digital.nhs.uk/media/20585/ IFS-Notice/pdf/IFSNotice.pdf (accessed February 2017).

27. World Health Organization, Division of Diarrhoeal and Acute Respiratory Disease Control (1991) Indicators for assessing breastfeeding practices. WHO/CDD/SER/91.14. http://apps.who.int/iris/bitstream/10665/62134/1/WHO_CDD_ SER_91.14.pdf (accessed February 2017).
28. Emilia-Romagna Regional Health Authority (2012) Emilia-Romagna regional health databases. http://salute. regione.emilia-romagna.it/siseps/sanita (accessed February 2017).

29. Magnusson M, Lagerberg D \& Wallby T (2016) No widening socioeconomic gap within a general decline in Swedish breastfeeding. Child Care Health Dev 42, 415-423.

30. Paolucci C, Gualanduzzi C, Finarelli AC et al. (2015) Coperture vaccinali nell'infanzia e nell'adolescenza. Anno 2014 (Data on infant immunisation coverage in 2015). http://salute.regione.emilia-romagna.it/sanita-pubblica/ documentazione/vaccinazioni (accessed February 2017). 